\title{
UGA-APS Soft X-ray Pilot Program at SER-CAT 22BM: \\ Phase II - Chromatic Exploration of Uncharted Aspects of Metals in Macromolecules
}

Bi-Cheng Wang, John Rose, John Chrzas, Lirong Chen, Palani Kandavelu, Dayong Zhou, Unmesh Chinte, Zheng-Qing Fu, Zhongmin Jin, James Fait, Gerold Rosenbaum, and Dennis Mills

University of Georgia, Athens, GA 30602, USA and

Advanced Photon Source, Argonne, IL, 60439, USA

Diffraction from the soft X-ray region (wavelength $>1.5 \AA$ ) has played an important role in recent success for macromolecular structure determinations by Native-SAD phasing (reviewed by Rose, Wang \& Weiss, 2015; Rose \& Wang, 2016). Other unique applications of soft X-rays for structural biology research, which may not be well recognized, are also becoming possible due to recent advances in both experimental design and data collection technology.

To make these new and unique applications of synchrotron soft X-rays available to the APS General User Community, a UGA-APS Pilot Program in this area was initiated in January 2016 at SER-CAT, Advanced Photon Source (APS), Argonne National Laboratory. Phase I of the Pilot Program was focused on fine-tuning the SER-CAT facility for Native-SAD that was reported at the 2016 ACA meeting in Denver. In this presentation, we are focusing on Phase II of the Pilot Program - the use of wavelength-dependent data for the chromatic exploration of uncharted biophysical/biochemical aspects of metals/ions in protein crystals. The theoretical basis and test results, as well as the procedures for accessing the Pilot Program through the General User portal, will be discussed. 